\title{
EDU Journal of Computer and Electrical Engineering
}

\section{Doctor Recommendation Based on Patient Syndrome Using Convolutional Neural Network}

\author{
Promila Haque $^{1 \times}$, Soumik Barua Pranto ${ }^{1}$, Sherra Adib Joha ${ }^{1}$ \\ ${ }^{1}$ Department of Computer, Science and Engineering, East Delta University, Abdullah Al Noman Road, Noman Socienty, East Nasirabad, \\ Khulshi, Chattagram 4209, Bangladesh
}

\author{
Received: November 19, 2021 \\ Revised: December 06, 2021 \\ Accepted: December 25, 2021 \\ Keywords \\ Health Recommendation System \\ Content-Based Filtering \\ $\mathrm{CNN}$
}

Discrete Rules

Deep Learning

\begin{abstract}
Recommendation systems in the online medical sector assist patients in finding appropriate doctors. This paper aimed to solve the complication in doctors' recommendations, concerning that people often struggle to see sure doctors according to their medical needs. Currently, most existing systems create doctors' recommendations through explicit or implicit feedback mechanisms. This doctor recommendation model does not depend on user feedback; instead, candidate doctors are generated for guidance solely from the user's current medical conditions. A prognosis is predicted for specific syndrome via CNN. By applying discrete rules, the system identifies and fetches the most relevant specialists according to the prediction and provides necessary information. The performance evaluation results of the proposed method are high and satisfactory.

(C) 2021 The authors. Published by EDU Journal of Computer and Electrical Engineering. This is an open access article under the CC BY NC license.
\end{abstract}

\section{INTRODUCTION}

At present, the health information needs of people are changing around the globe. Especially the Covid-19 pandemic situation has shown the importance of effective and accurate healthcare systems. In Bangladesh, a significant problem has been the difficulty and high cost of getting medical treatment for the past several years. Being an overpopulated and low economic infrastructure country, various kinds of illness spread rapidly. Moreover, the most prominent challenge in getting medical treatment for patients is finding suitable doctors. The proposed system provides results by analyzing the medical conditions given by the users to find out the appropriate doctors. The design follows the content-based recommendation using CNN for prediction and rule-based afterward for advice. The essential advantage of this system is that it does not rely on any previous user feedback. Instead, the proposed method can safeguard people by providing accurate and trustworthy suggestions of doctors. The structure of the paper is arranged as follows: section 2 is about related research, section 3 is about methodology, section 4 is about result analysis, and section 5 is about the conclusion.

\section{RELATED RESEARCH}

In recent years, recommender systems have grown in favor of effective online healthcare. Several researchers over the years have attempted to develop technologies to address the issues concerning recommendation processes. In addition, numerous methods have been developed to enhance the performance of recommendation systems. For example, traditional recommendation system methods include a recommendation based on collaborative filtering, content, demographic, association rules, network, etc. In [1], the association rules technique attempts to find the internal linkages between the userselected items, find which items are selected together, and are used in the following recommendation. In [2], the method uses demographic data to find similar users to complete the recommendation. In [3], the content-based way finds identical items based on the user's previous behavior or feedback and recommends items close to users' likes. The collaborative filtering approaches in [4], [5], [6] predict what a user would like based on ratings from other users who have similar tastes to the target user. In [7], the network-based method builds network models by using data on users' past preferences and selects new items that a given individual user might like.

Cheung et al. identified some critical issues resolved in the period ahead to make the recommendation systems more efficient [8]. The paper of Schafer et al. [9] addressed existing literature in the healthcare field for the last decade regarding recommender system utility. Hussein et al. [10] introduced a recommender system that uses various tree disease classification models to

\footnotetext{
${ }^{\boxplus}$ Corresponding author. E-mail address: promila@eastdelta.edu.bd (Promila Haque )

This work is licensed under a Creative Commons Attribution 4.0. License (CC BY NC 4.0)

Available online at http://edu-journals.com/index.php/ejcee

https://doi.org/ 10.46603/ejcee. $v 2 i 1.36$
} 
forecast the disease for a given patient. The focus was on condition, and there was no attempt to define any criteria or features by which a doctor will be evaluated for a specific patient. Wiesner and Pfeifer [11] presented a study to exploit several health recommender system challenges, concepts, and technical issues. Patients can use an online system to enter their medical problems into their proposed recommender system, and professionals who have enrolled with the system are in charge of disease recognition. However, no approach was taken for determining a suitable doctor for a particular patient. Narducci et al. [12] introduced a recommender system that leveraged the semantic association between a patient's symptoms and treatment to discover comparable patients. This system can suggest doctors with high ratings from patients, but it did not indicate any approach for assessing a patient's ratings to a particular doctor. Salunke, Kasar, and Smita presented a recommender system in [13] where a doctor profiler is constructed using natural language processing and user ratings. They mainly focused on creating recommendations depending on user ratings, but it was unclear exactly which type of factors is the patients rated the doctors on.

Blue and OPTUM Inc presented an article [14] that included methods to find patients with similar symptoms. After calculating the patient similarity metrics, subsequent patients were ranked, and recommendations were created for the top-ranked similar patients. Ceyhan, Orhan, and Domnori proposed a method in [15] that suggests medical tests based on a patient's symptoms and anamnesis by using minimal sequential optimization and Random Forest. Baofu Yu proposed a medical recommendation system by using BP neural network and SOM neural network [16]. The former applies content-based filtering and classifies the data by subject - information pages on the same topic are put in the same group. The latter one uses collaborative filtering and matches the model of users' interests with various groups of pages. Then the pages with greater values than a threshold are recommended to the patients. Almost all existing systems, on the whole, are focused on collecting doctor recommendations through direct feedback methods like ratings or reviews from past users. These systems are known as user-based recommender systems. But these user-based systems hold loopholes, and some essential concerns arise regarding the systems, such as: on which criteria are patients choosing a specific doctor, a patient's medical condition may not match completely with a previous user, the factors based on which users give their feedbacks are unknown and may vary from person to person, there might be no user feedback available on particular doctors, etc. Thus, investigating the issues above, instead of applying the commonly applied user Based methods, we went for a Rule-Based approach in our proposed work so that we do not need to depend on prior interaction of users with the system.

\section{METHODOLOGY}

Recommendation Systems are a tool that offers elements and communicant correspondence, communication, and information [17]. These are machine learning frameworks that assist clients with finding an item, and administrations Goldberg et al. built the first Recommendation System in 1992 [18] to help clients deal with an overabundance of communications in their mailbox. It is a filtering calculation in which clients collaborate by enrolling their responses to reports after reading them. In the most recent twenty years, the interest in a suggestion framework has just expanded. It permits clients to manage large amounts of information, giving them a choice of customized proposals, administrations, and substance. Subsequently, different procedures have been created and considered, both from the local scientific area and from organizations, since it permits them to build their profit [19]. Usually, a recommendation system is formed by two modules: database and filtering technique [20]. The data set is in charge of storing client information, items, and relevant appraisals. An algorithm generates the filtering technique, which is split into two steps. First, the similarity between clients or items is determined, resulting in a neighborhood for each item or customer. The system then predicts customer evaluations based on invisible things to the client, and only the best ones are used as suggestions [21].

The proposed system recommends doctors based on a patient's sickness symptoms, and since our inputs (signs) are onedimensional text data, we have used $1 \mathrm{D} C N N$ as a recommendation algorithm. The proposed system is connected into two parts. The first part uses a machine learning framework for disease prediction. The next part uses a rule-based approach to find the desired specialist for the predicted disease, thus recommending doctors follow the content-based recommender. The system requires an extensive dataset that contains a large number of symptoms where different combinations of symptoms lead to various diseases, and specialists for the predicted disease are found and recommended from the other two datasets of doctors. The total number of conditions included in the dataset was an aggregate of 261 and 500+ symptoms. By multiplying the dataset, every sickness symptom is gotten; a combination of the symptoms is made and added as new rows. After pre-preparing and augmentation, the dataset contains around 8835 rows with 489 unique signs. This was done to handle the issue of just having a solitary row for every disease, which brings about poor data training. This idea was motivated by this present reality situation where a patient, in any event, showing some of the symptoms of all the signs for a disease can be experiencing that sickness; accordingly. It is a consistent expansion of the dataset. The proposed methodology of the doctor recommendation system presented in this paper is shown in Figure 1.

\subsection{Symptom Collection}

Multiple symptoms of patients are taken as input and saved in CSV file format. The symptoms are taken in text format and converted into binary form. Then the symptoms are read and assigned binary values, as the machine cannot proceed and work with text data. For example, if a particular symptom is selected by a patient (present in a patient), it is given the value one, and not chosen symptoms are assigned the value 0 . This way, a testing set is created, matched with the training data set to reach a target value: the prognosis or disease.

\subsection{Symptom Filtering and Disease Prediction}

The primary dataset is divided through a split operation and grouped into two subsets for training and testing. This train-test- 
split method evaluates the machine learning algorithm's performance for the predictive modeling problem. The procedure involves taking the dataset and grouping it into two subsets. The initial subset utilized to fit the model is the training dataset. The test dataset is the second subset used to estimate the model's learning on new data. Thus, the work proceeds in two steps. First, the binary input values are converted to round figures in the training phase if there are any fractions. Then the prognosis names are given numerical values using the one-hot encoder because deep learning algorithms such as CNN cannot work directly with categorical data. A binary vector representation of categorical variables is known as one-hot encoding. Firstly the absolute values were converted to integer values. After that, a binary vector is created for each integer value, with all zero values save the integer's index, marked with a 1 . Then the particular amount of dataset selected for the training process is trained to predict which combination of symptoms refers to what prognosis.

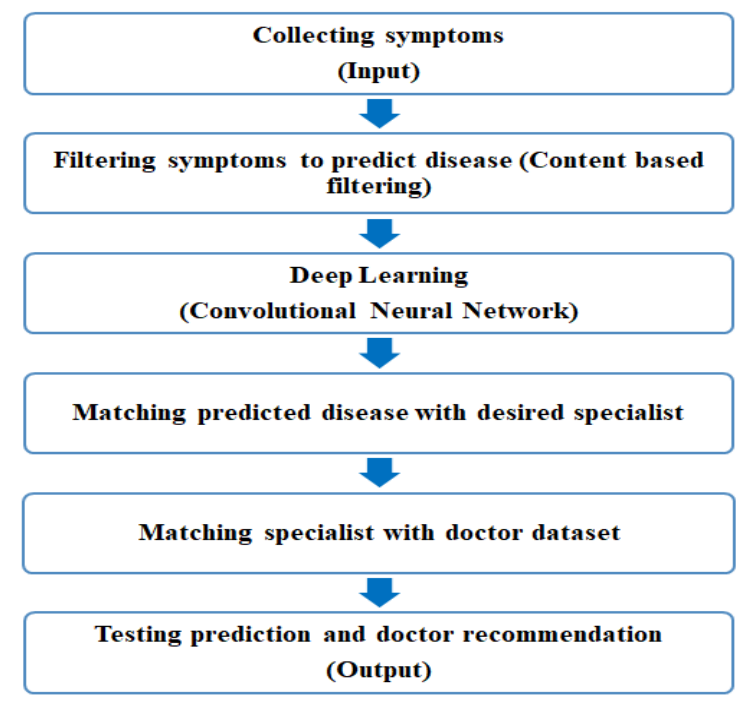

Figure 1. Framework of the proposed system.

The training is then followed by testing. Here, the selected testing dataset is used to test if the system can predict proper prognosis for various symptom combinations. For this, the input elements of the testing set are provided to the model without the outputs; the model looks for the closest possible match in the training set and gives predictions accordingly. Finally, the predicted result of the testing process is compared with the expected values to check the accuracy of the prediction. The prediction result is done by CNN (Convolutional Neural Network). For all of the convolution layer blocks, 1-D convolution was used. Finally, this network delivers autonomous signal classification through an end-to-end deep learning framework. Our proposed CNN model is shown in Table 1.

\subsection{Convolutional Neural Network}

Convolutional Neural Network (CNN) is widely used in deep learning. In this system, $\mathrm{CNN}$ has a vital role to play. A 1D convolutional neural network was used in this work, where it has been followed by the following steps of a convolutional neural network. In Figure 2, the simple architecture of the CNN model is shown. In Figure 3, the architecture of the deep learning process where prediction output is found after the softmax layer is displayed.

\subsubsection{Convolutional Layer}

The convolutional neural network $(\mathrm{CNN})$ is a specific kind of neural model that works with two-dimensional picture information, one-dimensional and three-dimensional information. In numerical terms, convolution is a function gotten from two given functions by coordination which communicates how the state is altered by the other. This layer plays out an operation called a "convolution".

Table 1. Proposed 1D CNN Model

\begin{tabular}{lll}
\hline Layer & Output Shape & Param \\
\hline conv1d_1 (Conv1D) & (None,132,160) & 480 \\
conv1d_2 (Conv1D) & (None,132,384) & 184704 \\
max_pooling1d_1(MaxPooling) & (None, 44, 384) & 0 \\
dropout_1 (Dropout) & (None, 44, 384) & 0 \\
conv1d_3 (Conv1D) & (None, 44, 384) & 442752 \\
max_pooling1d_2(MaxPooling) & (None, 22, 384) & 0 \\
dropout_2 (Dropout) & (None, 22, 384) & 0 \\
flatten_1 (Flatten) & (None, 8448) & 0 \\
dense_1 (Dense) & (None, 352) & 2974048 \\
dense_2(Dense) & (None, 1352) & 477256 \\
dense_3 (Dense) & (None, 160) & 216480 \\
dense_4 (Dense) & (None, 41) & 6601 \\
\hline
\end{tabular}

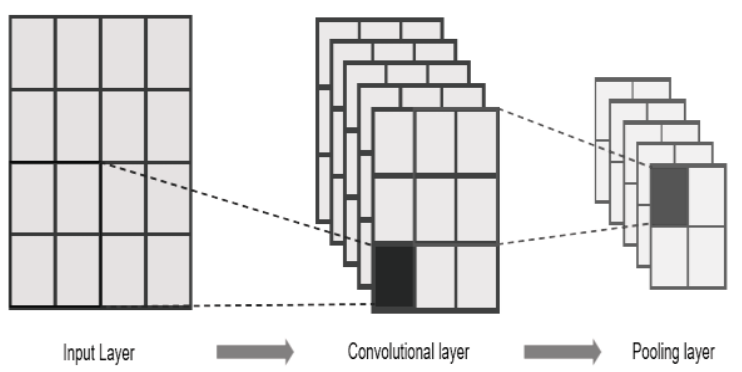

Figure 2. Simple CNN Architecture.

Convolution is a linear operation that comprises the multiplication of weights with the input in a convolutional neural network, similar to a standard neural network. The expansion is conducted between various information and a two-dimensional array of importance, termed a kernel, because the process was designed for twodimensional data. A single value is produced by multiplying the filter with the array once. As the filter is applied on numerous occasions to the input array, the outcome is a two-dimensional cluster of output values that address the filtering of the information. Accordingly, the two-dimensional output array from this activity is known as a "feature map" [22], shown in Figure 4. When a feature map is made, we can pass each value in the feature map through a nonlinearity, like a ReLU, similar to the output of a fully connected layer. In our work, we defined a one-dimensional input that few elements, all with the estimation of 0.0 , with two parts, knock in the 
center with the values 1,0 . The information to Keras ought to be three-dimensional for a 1D convolutional layer [23].

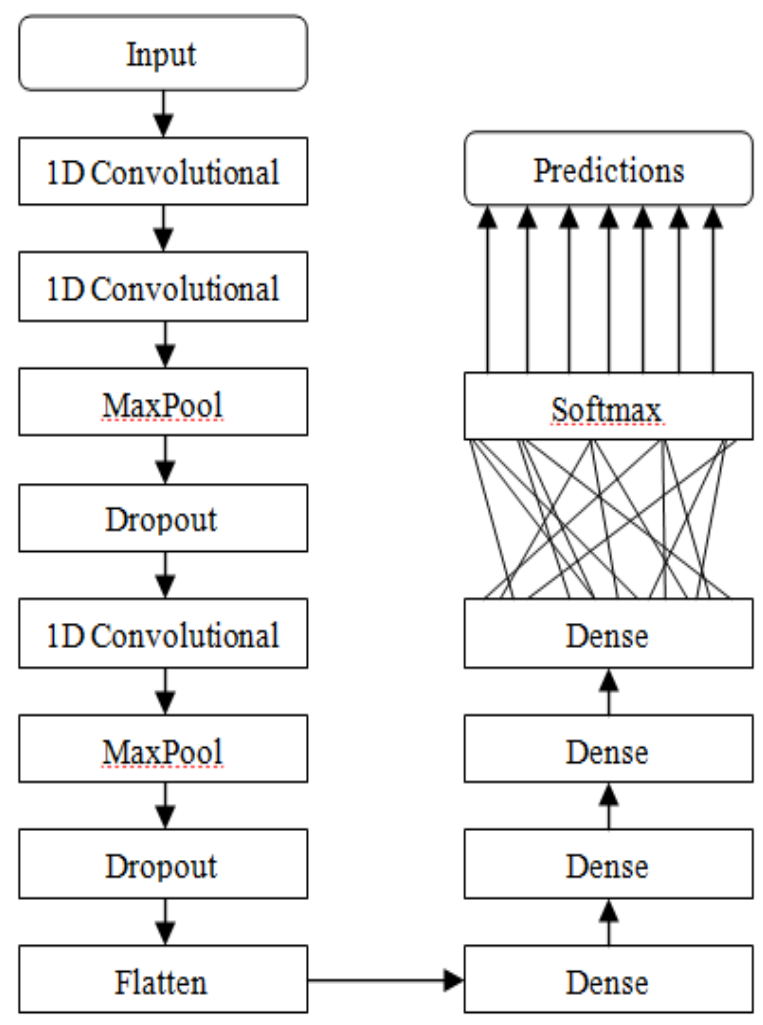

Figure 3. The architecture of the proposed $\mathrm{CNN}$ layer by layer model.

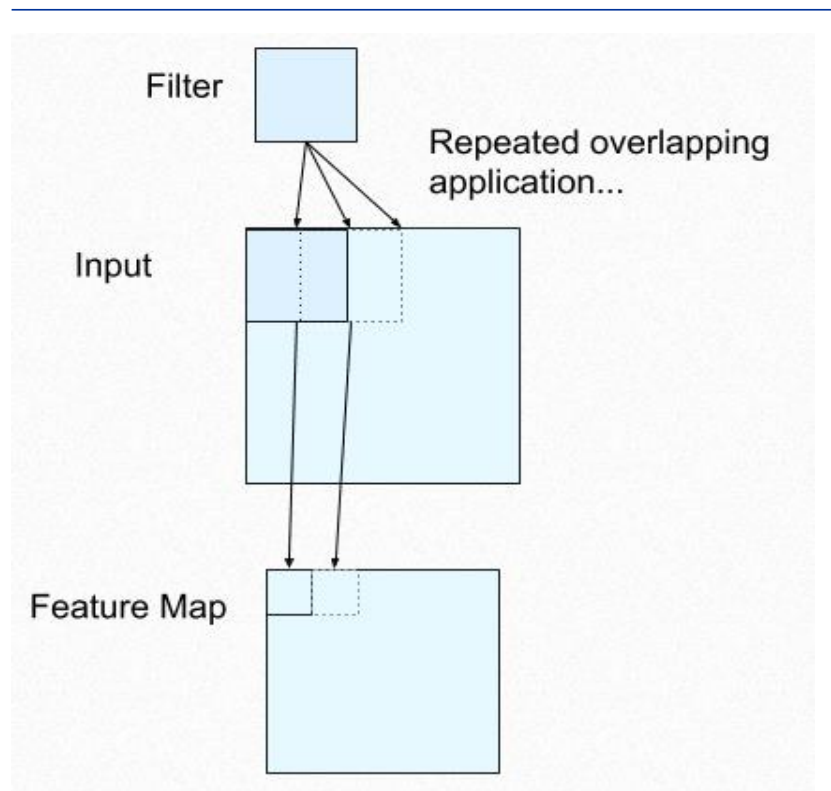

Figure 4. Convolution layer.

\subsubsection{ReLU layer}

The Rectified Linear Unit, or ReLU, is not a different part of the process of convolutional neural networks [30]. It's a helpful improvement to the convolution activity since it functions as an activation function, assuring nonlinearity as data passes through each network layer. Without it, the data supplied into each layer would lose its dimensionality, which we wanted to keep.

\subsubsection{Pooling layer}

Another CNN layer that downsamples a feature map reduces the sample size. This also expedites the process. A pooled feature map results from this, which decreases the number of parameters of the network must process. Figure 5 depicts the pooling layer.

\subsubsection{Max Pooling Layer}

After each convolutional layer, it's common to add a type of activity to CNNs. When max pooling is employed in a model, the amount of pixels in the previous convolutional layer's output is reduced. Hence, lowering the dimensionality of images [24]. It's a part of the pooling layer, and we employed it since the maxpooling layer takes the maximum input of a convolved feature to make things easier. The final machine learning architecture of our model is shown in Figure 6.

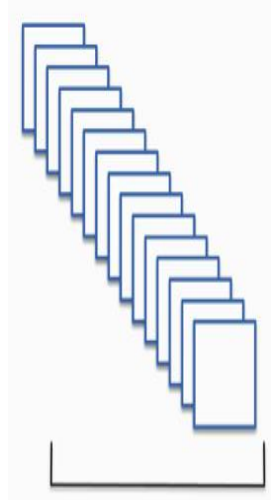

Pooling Layer

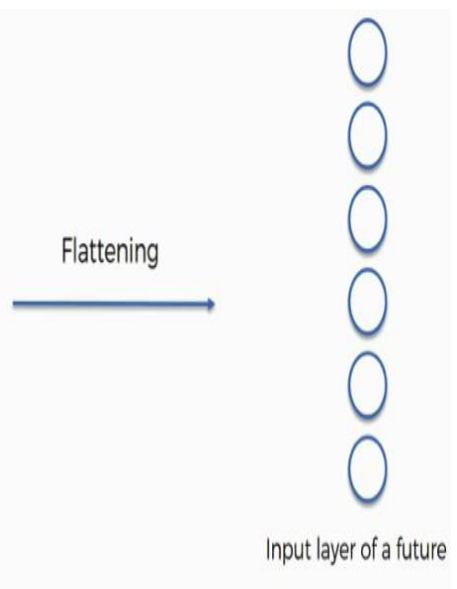

Figure 5. Pooling layer.

\subsection{Disease Prediction}

After filtering the symptoms through a machine learning algorithm, we found a predicted disease. As we expected the disease, might not be $100 \%$ accurate. But the actual condition should be very close to the predicted one. And the actual illness can be treated by the doctors under the category of the expected disease. So instead of directly suggesting doctors for the exact expected condition, we called the type of the expected disease to offer doctors under that category. For this purpose, we used the rule-based system to determine under which category the indicated disease falls in with the help of a dataset that establishes a connection between diseases and specialist type.

\subsection{Fetching Doctor's Details}

After determining the specialist category for the predicted disease, doctors under that category are fetched from another dataset linked through the category attribute. The dataset contains all the necessary information of the doctors. Details of a doctor consist of their qualifications, work institution, chamber, location, contact number, etc.

\subsection{Doctor's Recommendation}

Finally, as the output of the doctor recommendation system, the result is displayed in a chart where various desired doctors for the given medical condition are shown. The doctor's details include the doctor's full name, educational information to evaluate their quality, the institution they are currently working under, 
chamber, detailed location to help users know which is closer to them, and contact number to set an appointment. Patients can select the doctor according to preference and convenience based on this information.
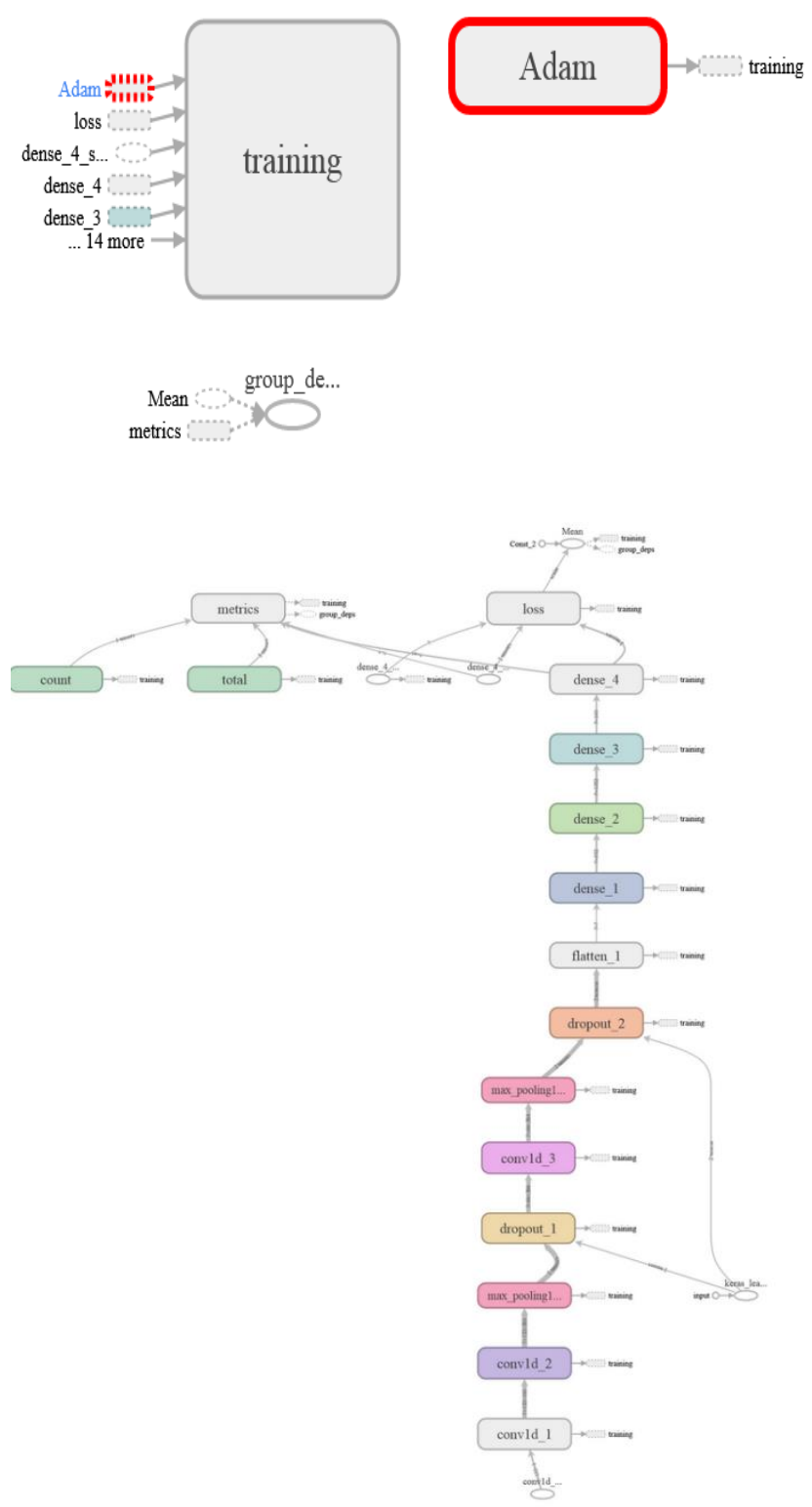

Figure 6. Complete CNN Architecture.

\section{RESULT ANALYSIS}

Our proposed model has been implemented using Python programming language [25] along with Tensor Flow [26], Keras [27], Matplotlib [28]. Three datasets have been used and used sequentially to complete the whole process in this model. At first, a large dataset containing 134 types of symptoms and 52 targeted prognoses makes up a vast number of combinations and leads to various types of prediction [29]. The dataset is divided into training and testing data, accounting for $60 \%$ of the dataset and testing data accounting for $40 \%$. Figure 7 depicts a sample of the training and testing dataset. A dataset was established to detect the relationships between prognosis and their corresponding specialist category - a dataset containing all doctors and their class and other necessary information. The desired doctors are fetched along with the required information using the category attribute [30]. After completing the proposed work procedure, we tested the model with various possible inputs and printed the output into text viewable format. A sample of output is shown in Figure 8 . The classification result of the proposed model can be analyzed and evaluated through a confusion matrix, which explains how capable the system is of providing accurate results. Finally, the accuracy, precision, recall, F1-score value, and the confusion matrix [31] graph are given in Table 2 and Figure 9.

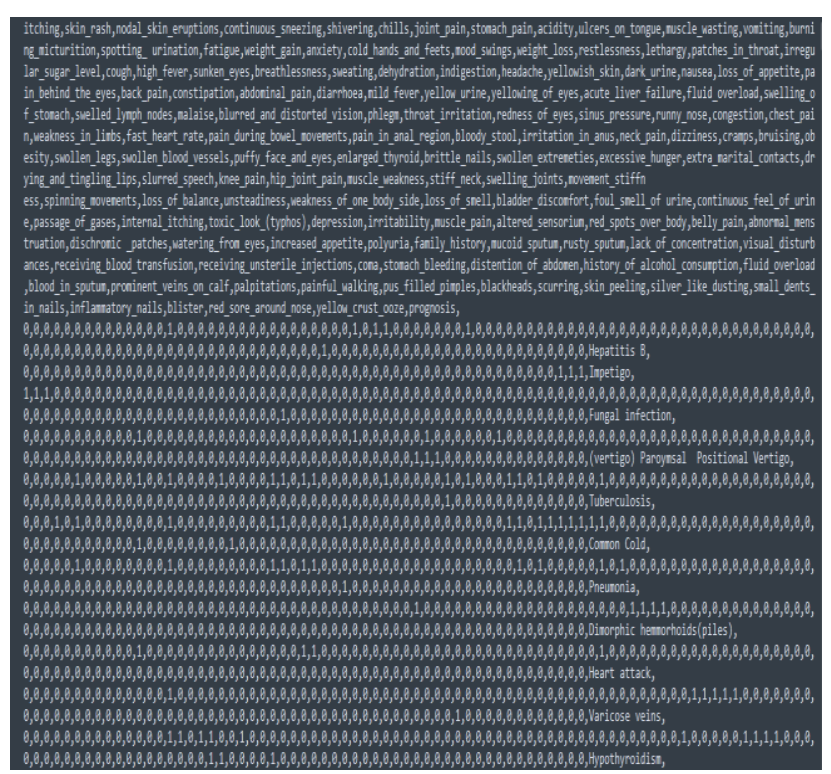

Figure 7. Sample of training and testing dataset.

Disease Doc_name $\quad$ Address Fungal inf Prof. Dr. Mansurul Alam 19, K.B. Fazlul 031656481 Fungal inf Prof. Dr. Mansurul Alam 19, K.B. Fazlul o 031656481 Fungal inf Prof. Dr. Mansurul Alam 19, K.B. Fazlul 031656481 Urinary tr: Dr. Md. Mizanur Rahman 19, K.B. Fazlul o31656481 Psoriasis Prof. Dr. Mansurul Alam 19, K.B. Fazlul 031656481 Impetigo Prof. Dr. Mansurul Alam 19, K.B. Fazlul o 031656481 Fungal inf Prof. Dr. Mansurul Alam 19, K.B. Fazlul 031656481 Chronic ch Dr. Shamim Boksha 14/15, Dampar 031627033 Drug Reac Dr. Abdullah Al-Mamun 94/103, Katalgê 1976021453 Peptic ulc Dr. Shamim Boksha 14/15, Dampar 031627033 AIDS Dr. Shamim Ara (Siju) 94/103, Katalgẹ 1825781210

Figure 8. Sample output of recommended doctors for the particular patient syndrome.

Table 2. Performance analysis table

\begin{tabular}{llll}
\hline CNN & Precision & Recall & F1 Score \\
\hline Accuracy & & & 0.96 \\
Macro average & 0.80 & 0.81 & 0.80 \\
Weighted average & 0.95 & 0.96 & 0.95 \\
\hline
\end{tabular}

The accuracy result during the testing and training phase is visible in Figure 10 and Figure 11. The X-axis indicates the number of epochs, and the Y-axis represents the training-testing models' 
accuracy gain. Various algorithms were applied for the proposed doctor recommendation system to identify which one gives better results for the selected approach. The applied algorithms include 1D Convolutional Neural Network, Artificial Neural Network, Decision Tree, Random Forest, and Extreme Gradient Boosting.

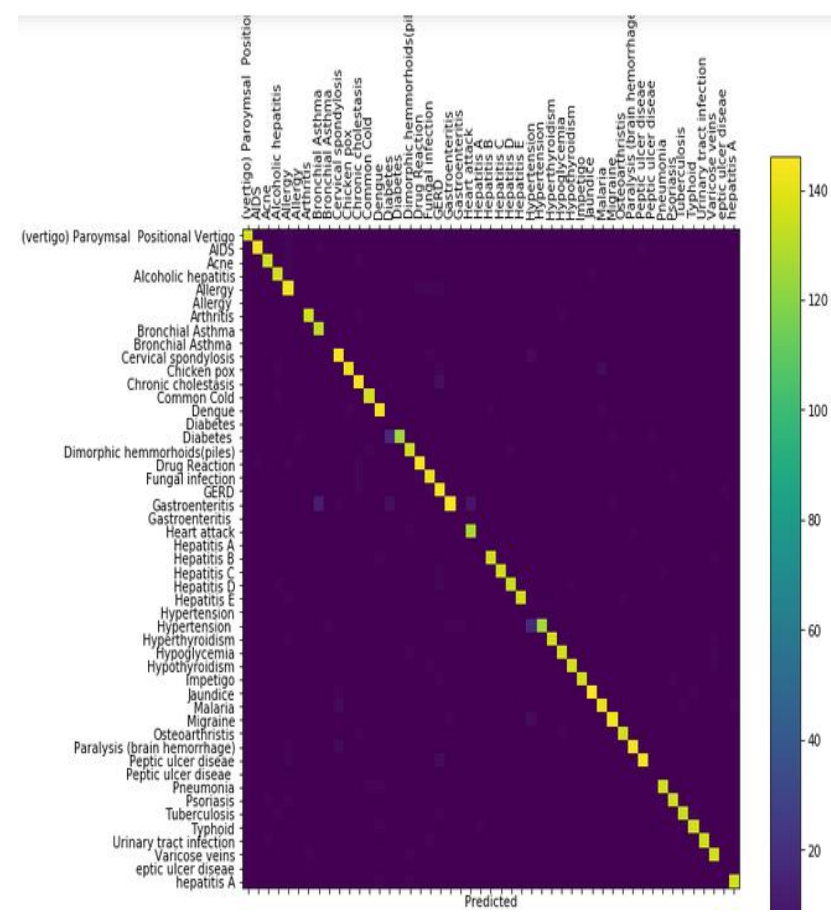

Figure 9. Confusion matrix prediction graph.

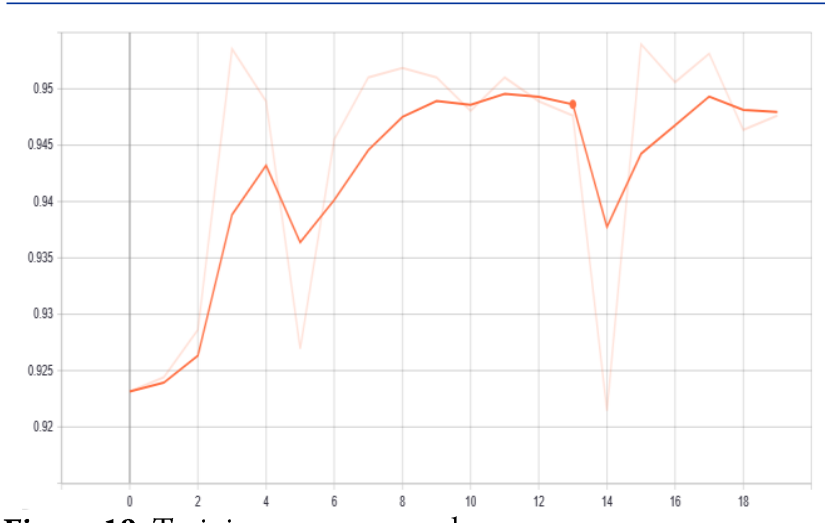

Figure 10. Training accuracy graph.

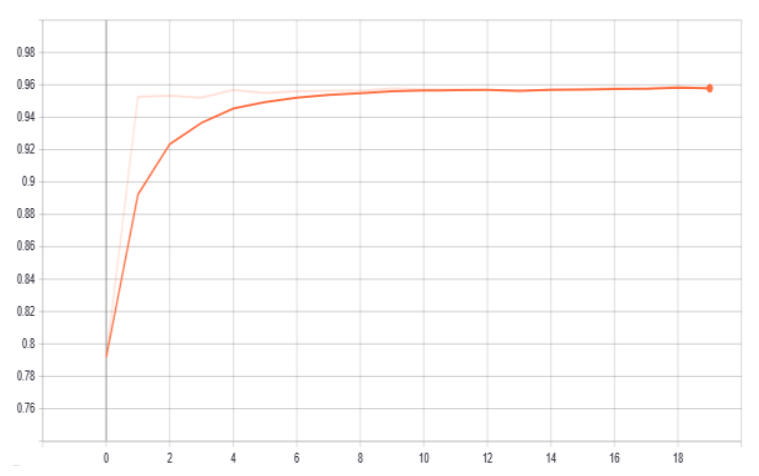

Figure 11. Testing accuracy graph.
From the comparison shown in Figure 12, we can conclude that $\mathrm{CNN}$ is superior to the other algorithms for this case as it gives the highest accuracy percentage among all.

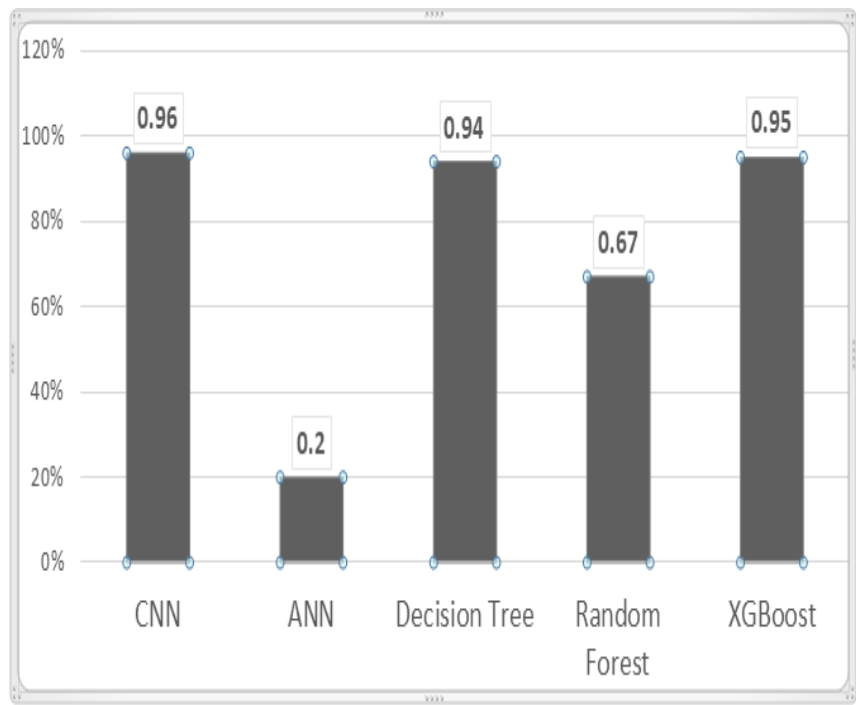

Figure 12. Performance analysis of different algorithms.

\section{CONCLUSIONS}

Doctor recommendation systems are of the essence for solving the issue of information overload that appears on online healthcare platforms. Patients can use technology to swiftly and accurately filter out many irrelevant doctors and identify doctors that fit their specific needs, allowing them to receive valuable personalized online healthcare services. This research proposed a personalized content-based doctor recommendation system using Neural Network Algorithm, rule inference, and various approaches for locating doctors that best meet patients' needs based on information gleaned from their symptoms. Rather than relying on prior interaction of users with the system as done in existing recommenders, the proposed method uses $\mathrm{CNN}$ for prediction and constructs rules to link the user's medical conditions with specialists and generate candidate doctors for a recommendation. The results obtained from the proposed system were excellent and satisfactory. According to the results, this method can provide an appropriate recommendation of doctors for particular medical conditions with an accuracy of $96 \%$. The proposed way of recommendation is straightforward, reliable, easy to use. It could be easily incorporated into various healthcare systems to enhance the quality of doctor recommendations. We plan to incorporate a user interface into our proposed system to make it more functional in the future. We also plan to enable independent user input. As of now, users have to select from the given limited symptoms. So to overcome this limitation, we want to allow users to provide new inputs of their own. The system can further be improved by incorporating various other symptoms and diseases and increasing the number of cases for training and testing. Moreover, we can include various filters for recommendation generation, such as location-based, fees-based, review-based, etc. Users can choose based on which criteria they prefer the doctors to be suggested. Additionally, 
some home remedies or primary treatments can be recommended. Finally, and most importantly, we plan to incorporate new techniques such as Fuzzy logic into the system to enhance accuracy.

\section{REFERENCES}

[1] S. J. Gong, "Personalized recommendation system based on association rules mining and collaborative filtering", Applied Mechanics and Materials, Vol. 39. Trans Tech Publications Ltd, 2011.

[2] L. Chen, R. Nayak, and Y. Xu, "A recommendation method for online dating networks based on social relations and demographic information." 2011 International Conference on Advances in Social Networks Analysis and Mining. IEEE, 2011.

[3] M. Kompan, and M. Bieliková, "Content-based news recommendation." International conference on electronic commerce and web technologies. Springer, Berlin, Heidelberg, 2010.

[4] A.L.Deng, Y.Y.Zhu, and B. Shi, "A collaborative filtering recommendation algorithm based on item rating prediction," Journal of Software, vol.14, no. 9, pp. 1621-1628, 2003.

[5] C. Huang, J. Yin, and J. Wang. "Collaborative filtering recommendation algorithm for uncertain nearest neighbor," Journal of Computer Science, vol. 33, no. 8, pp. 1369-1377, 2010.

[6] J. Bobadilla, A. Hernando, F. Ortega, and J. Bernal, 2011. A framework for collaborative filtering recommender systems. Expert Systems with Applications, vol. 38, no. 12, pp.14609-14623, 2011.

[7] K. Chen, P. Han, J. Wu, "Recommendation algorithm for heterogeneous social networks based on user clustering," Journal of Computer Science, vol. 36, no. 2, pp. 2-5,(Chinese), 2013.

[8] K. L. Cheung, D. Durusu, X. Sui, and H. de Vries, "How Recommender Systems Could Support and Enhance Computer-Tailored Digital Health Programs: A Scoping Review." Digital Health, vol. 5, pp. 1-19, 2019.

[9] H. Schafer, S. Hor-Fraile, and R. P. Kumar, A. C. Valdez, A. Said, H. Torkamaan, T. Ulmer and C. Trattner. "Towards health (aware) recommender systems," In Proceedings of the International conference on digital health, pp. 157-161,2017, London.

[10] A. S. Hussein, W. M. Omar, X. Li and M. Ati, "Efficient Chronic Disease Diagnosis Prediction and Recommendation System." In IEEE-EMBS Conference on Biomedical Engineering and Sciences, pp. 209-214, IEEE, 2012, doi:10.1109/IECBES.2012.6498117.

[11] M. Wiesner and D. Pfeifer, "Adapting Recommender Systems to the Requirements of Personal Health Record Systems." In Proceedings of the 1st ACM International Health Informatics Symposium, pp.410-414, 2010. doi:10.1145/1882992.1883053.

[12] F. Narducci, C. Musto, M. Polignano, M. de Gemmis, P. Lops and G. Semeraro, "A Recommender System for $14 \mathrm{M}$. WAQAR ET AL. Connecting Patients to the Right Doctors in the Health Net Social Network." In Proceedings of the 24th International Conference on World Wide Web, pp. 81-82, 2015. doi:10.1145/2740908.2742748.

[13] A. Salunke, B. Kasar, and L. Smita,"Personalized Recommendation System for Medical Assistance Using Hybrid Filtering," International Journal of Computer Applications, vol.128, no. 9, pp. 6-10, 2015.

[14] J.Blue,"Healthcare similarity engine," U.S. Patent No. 10,127,359. 13 Nov. 2018.

[15] C. Migena, Z. Orhan, and E. Domnori. "e-Medical Test Recommendation System Based on the Analysis of Patients' Symptoms and Anamneses," Part of the IFMBE Proceedings book series, vol. 62, March 15, 2017.

[16] W. Chen, and M. Xu, "The research of doctors recommendation algorithm based on clustering and collaborative filtering," Proceedings of the 23rd International Conference on Industrial Engineering and Engineering Management, Atlantis Press, Paris, 2017.

[17] J. Bobadilla, F. Serradilla, and A. Hernando,"Collaborative filtering adapted to recommender systems of e-learning," Knowledge-Based Systems, vol. 22, no. 4, pp. $261-265,2009$.
[18] D. Goldberg, D. Nichols, B. M. Oki, and D. Terry, "Using collaborative filtering to weave an information tapestry," Commun. ACM, vol.35, pp.6170, 1992 .

[19] J. Bobadilla, F. Ortega, A. Hernando, and A. Guti'errez, "Recommender systems survey," Knowledge-Based Systems, vol. 46, pp. 109 - 132, 2013.

[20] G. Adomavicius and A. Tuzhilin, "Toward the next generation of recommender systems: a survey of the state-of-the-art and possible extensions," IEEE Transactions on Knowledge and Data Engineering, vol. 17, no. 6, pp. 734-749, 2005.

[21] X. Zhou, Y. Xu, Y. Li, A. Josang, and C. Cox, "The state-of-the-art in personalized recommender systems for social networking," Artificial Intelligence Review, vol. 37, no. 2, pp. 119-132, 2012.

[22] T. T. Contributors, "What is convolutional neural network? - Definition from WhatIs.com," Search Enterprise AI, 26-Apr-2018. [Online]. Available:

https://searchenterpriseai.techtarget.com/definition/convolutionalneural-network. [Accessed: 24Jan-2021].

[23] "Keras Conv1D: Working with 1D Convolutional Neural Networks in Keras," MissingLink.ai. [Online]. Available: https://missinglink.ai/guides/keras/keras-conv1d-working-1dconvolutional-neural-networks-keras/. [Accessed: 24Jan-2021].

[24] "Max Pooling in Convolutional Neural Networks explained," deeplizard. [Online]. Available: https://deeplizard.com/learn/video/ZjM_XQa5s6s. [Accessed: 31-Mar-2021].

[25] "Top 10 Reasons Why Python is So Popular With Developers in 2021," upGrad blog, 03-Apr-2021. [Online]. Available: https://www.upgrad.com/blog/reasons-why-python-popular-withdevelopers/. [Accessed: 09-May-2021].

[26] Simplilearn, "What is Tensorflow: Deep Learning Libraries and Program Elements Explained," Simplilearn.com, 24Mar-2021. [Online]. Available: https://www.simplilearn.com/tutorials/deep-learning-tutorial/what-istensorflow. [Accessed: 09-May-2021].

[27] J. Brownlee, "Your First Deep Learning Project in Python with Keras StepBy-Step," Machine Learning Mastery, 15-Sep-2020. [Online]. Available: https://machinelearningmastery.com/tutorial-first-neural-networkpython-keras/. [Accessed: 09-May-2021].

[28] R. M, "What Is Matplotlib In Python? How to use it for plotting?," ActiveState, 08-Apr-2021. [Online]. Available: https://www.activestate.com/resources/quick-reads/what-is-matplotlibin-python-how-to-use-it-for-plotting/. [Accessed: 09-May-2021].

[29] anujdutt9, "anujdutt9/Disease-Prediction-from-Symptoms," GitHub. [Online]. Available: https://github.com/anujdutt9/Disease-Predictionfrom-Symptoms/blob/master/dataset/test_data.csv. [Accessed: 09-May2021].

[30] "Specialist Doctor List in Chittagong - Doctors in Chattogram," Doctor Bangladesh, 05-Apr-2021. [Online]. Available: https://www.doctorbangladesh.com/doctors-chittagong/. [Accessed: 22Apr-2021].

[31] "Confusion matrix in machine learning," 21-Aug-2020. [Online]. Available: https://www.geeksforgeeks.org/confusion-matrix-machine-learning/. [Accessed: 03-May-2021].

[32] L. Sulieman, D. Gilmore, C. French, R. Cronin, G. Jackson, M. Russell and D. Fabbri, "Classifying Patient Portal Messages Using Convolutional Neural Networks," Journal of Biomedical Informatics, vol. 74, pp. 59-70, 2017. Doi: 10.1016/j.jbi.2017.08.014.

[33] C. Yao, Y. Qu, L. Guo, L. Chao, W. Cui, and L. Feng," A Convolutional Neural Network Model for Online Medical Guidance", IEEE Access, vol.4, pp. 4094-4103, 2016. Doi:10.1109/ACCESS.2016.2594839. 